\title{
Hybrid Biodegradable Nanomotors through Compartmentalized Synthesis
}

Imke A. B. Pijpers, Shoupeng Cao, Antoni Llopis-Lorente, Jianzhi Zhu, Shidong Song, Rick R. M. Joosten, Fenghua Meng, Heiner Friedrich, David S. Williams, Samuel Sánchez, Jan C. M. van Hest, * and Loai K. E. A. Abdelmohsen*

Cite This: Nano Lett. 2020, 20, 4472-4480

Read Online

ACCESS | 네 Metrics \& More | 回 Article Recommendations | sl Supporting Information

ABSTRACT: Designer particles that are embued with nanomachinery for autonomous motion have great potential for biomedical applications; however, their development is highly demanding with respect to biodegradability/compatibility. Previously, biodegradable propulsive machinery based on enzymes has been presented. However, enzymes are highly susceptible to proteolysis and deactivation in biological milieu. Biodegradable hybrid nanomotors powered by catalytic inorganic nanoparticles provide a proteolytically stable alternative to those based upon

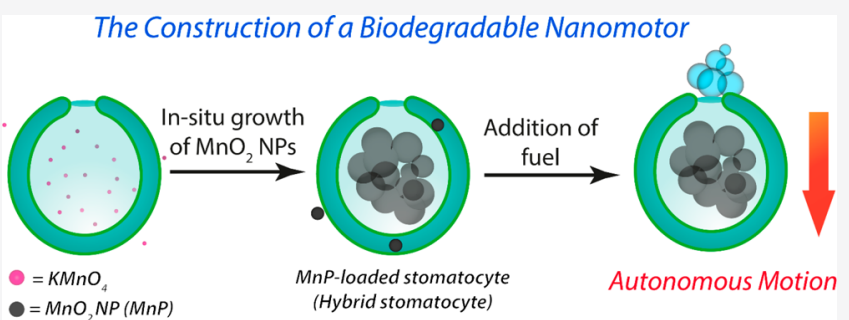
enzymes. Herein we describe the assembly of hybrid biodegradable nanomotors capable of transducing chemical energy into motion. Such nanomotors are constructed through a process of compartmentalized synthesis of inorganic $\mathrm{MnO}_{2}$ nanoparticles ( $\mathrm{MnPs}$ ) within the cavity of organic stomatocytes. We show that the nanomotors remain active in cellular environments and do not compromise cell viability. Effective tumor penetration of hybrid nanomotors is also demonstrated in proof-of-principle experiments. Overall, this work represents a new prospect for engineering of nanomotors that can retain their functionality within biological contexts.

KEYWORDS: Biodegradable nanomotors, Compartmentalization, Stomatocyte, Hybrid nanosystems, Biomedical applications

$\mathrm{E}$ ngineering of motile vehicles at the micro- and nanoscale with built-in systems of chemical propulsion has witnessed a surge of interest over the past decade. ${ }^{1-4}$ In such systems, autonomous motion is achieved by the conversion of chemical energy into kinetic energy. ${ }^{5,6}$ Such chemical processes are empowered by various forms of bio-organic and inorganic catalysts (e.g., enzymes and platinum nanoparticles). ${ }^{7,8}$ The potential of motile materials in biomedical research (by, e.g., facilitating the active transport of biofunctional cargo) has been presented as a key opportunity for the exploitation of this technology. ${ }^{9-11}$ Indeed, developing robust chemistries that can give rise to motility and diverse structural architectures that form the molecular chassis for these vehicles drives fascinating progress toward the actual translation of these systems. ${ }^{12-15}$ In particular, controlling physical properties such as particle morphology and composition is essential to expand the motors' potential in applications that can benefit from nonBrownian motility. ${ }^{16-19}$ Developing micro- and nanomotors suitable for biomedical integration has been explored by various research groups, who have demonstrated motors' capability in undertaking complex tasks. ${ }^{20-23}$ Städler et al. showed the capacity of microswimmers for directed penetration of three-dimensional (3D) cell spheroids, offering a wealth of opportunities for the application of micro- and nanomotors in tissue penetration and cargo/drug delivery. ${ }^{24}$
Recently, the group of Sen showed the ability of liposome protocells to undergo positive and negative chemotaxis behavior, unlocking tremendous opportunities for biological transport. ${ }^{25}$ Such systems not only demonstrate the advantageous aspects of non-Brownian motility within biological contexts but also highlight the challenges faced in engineering of new features for such complex materials and emphasize the importance of molecular interactions between the motors and their environment-a significant aspect for their effective function and application.

Miniaturization of motile systems to the nanoscale is extremely challenging, but it is critical for their development toward biomedical applications by facilitating effective interactions with cells and tissues while avoiding the unfavorable fate of microscopic particles (e.g., poor circulation and distribution). ${ }^{26,27}$ Developing both the nanomotor chassis and propulsive unit for biodegradability and compatibility

Received: March 23, 2020

Revised: May 19, 2020

Published: May 19, 2020 


\section{A. Self-Assembly and Shape Transformation of Polymersomes}

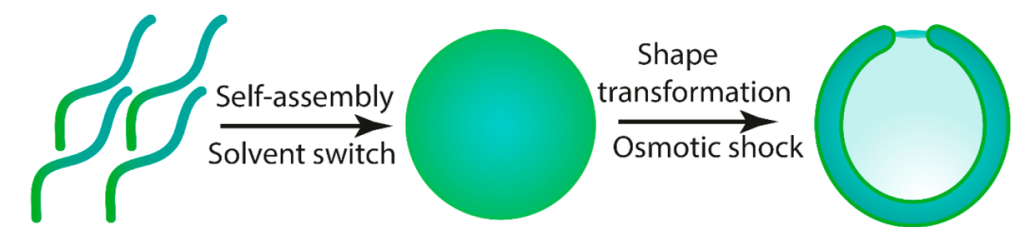

Block co-polymers

Spherical polymersome

Bowl-shaped stomatocyte

B. Compartmentalized Synthesis of Catalytic Machinery

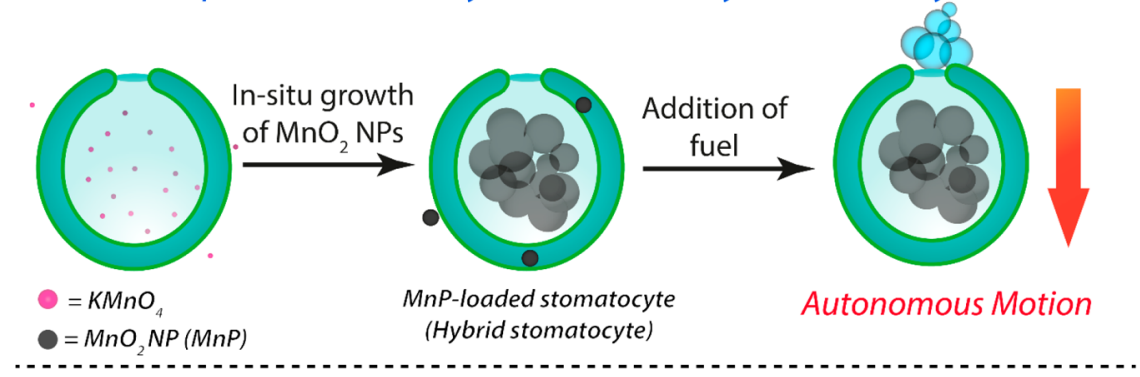

Transmission Electron Microscopy \& Tomography
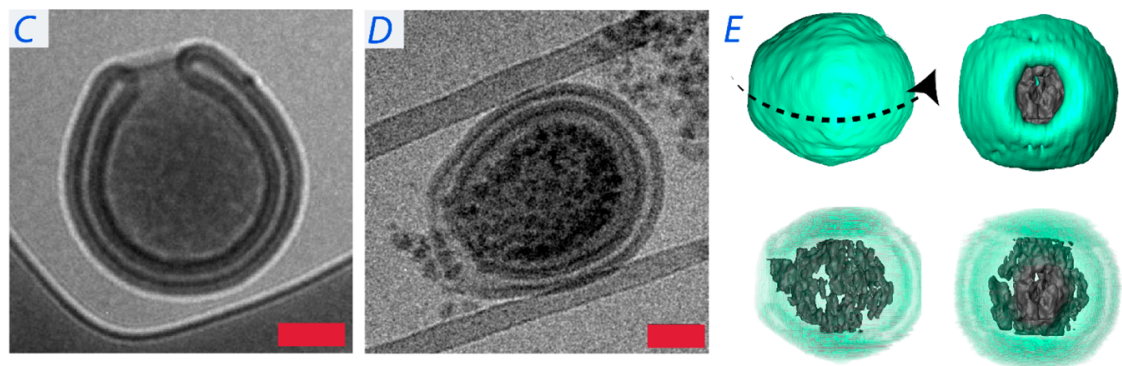

Figure 1. (A) Schematic demonstrating the formation of spherical polymersomes and their subsequent osmotic-induced shape transformation into stomatocytes. (B) Schematic demonstrating the compartmentalized synthesis of MnPs within the stomatocytes' lumen and subsequent formation of hybrid nanomotors that can convert fuel into mechanical motion. (C) Cryo-TEM image of an empty stomatocyte. (D) Cryo-TEM image of a MnP-loaded stomatocyte. (E) 3D rendering of a MnP-loaded stomatocyte obtained by TEM tomography. Scale bars $=200 \mathrm{~nm}$.

while preventing unwanted deactivation by inevitable environmental factors (e.g., the presence of proteolytic enzymes) is an important milestone toward the widespread adoption of such materials. Dual-compartmentalized stomatocytes, which are bowl-shaped polymersomes, ${ }^{28,29}$ are uniquely suited for nanomotor fabrication because of their asymmetric structure with an internal cavity (or stomach) that is in direct contact with the external environment via a tunable neck. In our previous work, we have showcased the potential of such stomatocytes to function as nanomotors by encapsulating enzymatic cargo to drive their mechanical motion; ${ }^{30,31}$ however, that work utilized nondegradable polystyrene block copolymers (BCPs) that, without doubt, lack any immediate biomedical relevance. The motility of such systems using enzymatic cargo was achieved by modulating the size of the opening (or neck) during encapsulation so that the macromolecular cargo could be effectively sequestered, preventing unwanted release or digestion by external proteases. From that work, the capacity of stomatocytes to confine and protect biocatalysts in their stomach, allowing enzymatic reactions to take place that can drive motility in complex biological media, was apparent. Capitalizing on that research, our group has developed biodegradable stomatocytes by employing welldefined poly(ethylene glycol)-block-poly(D,L-lactide) (PEGPDLLA) BCPs. ${ }^{32,33}$ Such PDLLA-based stomatocytes were effectively integrated into biomedical research through, for example, incorporation of photosensitizers and surface coating with cell membrane components. ${ }^{34}$ On the basis of these developments, biodegradable stomatocyte nanomotors with enzymes covalently tethered in their cavity have been reported. ${ }^{35}$ However, as the neck size of these motors was not controlled, the protection capacity was lost, leaving incorporated enzymes exposed to proteolytic deactivationseverely limiting, if not withholding, their application in biological contexts.

Building on these recent developments and the considerable capacity of stomatosomal architectures, exploiting the unique structure of PDLLA stomatocytes as nanomotors that can function within biological contexts holds great future potential for biomedical applications. To this end, in the present work we employed inorganic nanoparticles as robust catalytic nanomachinery that can drive the stomatocytes' thrust in harsh proteolytic media. Inorganic catalysts, such as manganese dioxide $\left(\mathrm{MnO}_{2}\right)$, have found application in, for example, cancer therapy. ${ }^{36-38}$ In general, such nanoparticles are generated through surface stabilization or in situ formation using protein or polymer nanostructures, ${ }^{39,40}$ making hybrid architectures. As $\mathrm{MnO}_{2}$ is capable of catalase-mimetic catalytic decomposition of hydrogen peroxide $\left(\mathrm{H}_{2} \mathrm{O}_{2}\right)$ into water and oxygen (to drive motion through nanobubble formation), it is a suitable candidate to replace enzymes as the propulsive units for our biodegradable/compatible stomatocyte nanomotors. 
Furthermore, the utility of $\mathrm{MnO}_{2}$ particles as contrast agents has recently been shown through in vivo studies. ${ }^{41}$ In fact, researchers have recently pointed out the broad potential of enzyme-like inorganic particles for biomedical applications, as they hold interesting advantages: higher catalytic stability, ease of modification/functionalization, lower manufacturing costs compared with enzymes, utility as multimodal platforms, and remote control by external cues (magnetic fields, light, etc.). ${ }^{42,43}$ In this paper, we demonstrate the ability of PDLLA stomatocytes to be used for the compartmentalized synthesis of $\mathrm{MnO}_{2}$, a strategy that results in highly effective functional integration that yields fully biodegradable and biocompatible nanomotors that are not vulnerable to enzymatic deactivation in complex biological media. Using advanced characterization techniques, we explore the structure of hybrid nanomotors and demonstrate their enhanced motion, stability against protease deactivation, degradability, and compatibility with cells. In this way, this work demonstrates a novel concept for the design principles of a nanomotor platform that is primed for further application in biomedical research. In doing so, we also showcase a robust approach for the synthesis of inorganic catalysts using a compartmentalization approach to support the fabrication of complex, multifunctional nanoarchitectures.

Stomatocyte nanomotors were assembled from the bottomup utilizing biodegradable copolymers comprising 1 and $2 \mathrm{kDa}$ PEG and PDLLA $(n=95)$. Such copolymers were synthesized via organo-catalyzed ring-opening polymerization following our previously published procedure (Supporting Information (SI) section S2, Figures S1 and S2, and Table S1). ${ }^{33}$ Solventinduced supramolecular self-assembly of amphiphilic PEGPDLLA into polymersomes was performed using equimolar blends of both copolymers in organic solvent (10 wt \% in THF/dioxane, 1:4 v/v) followed by controlled addition of an equal volume of Milli-Q water. The shape transformation of spherical polymersomes into bowl-shaped stomatocytes was then achieved by applying an osmotic pressure on the plasticized membrane through dialysis against $75 \mathrm{mM} \mathrm{NaCl}$ at $4{ }^{\circ} \mathrm{C}$ (Figure $1 \mathrm{~A}, \mathrm{C}$ ). Tailoring the salt concentration during dialysis can, to a certain extent, tune the neck size of such stomatocytes through increased deflation and consequent volume reduction. Under the conditions employed, an average neck size of $94 \pm 50 \mathrm{~nm}$ was achieved; this is ca. 15 times larger than the size of a protein like bovine serum albumin (BSA). The formation of such stomatocytes was monitored and confirmed using dynamic light scattering (DLS), nanotracking analysis (NTA), and cryogenic transmission electron microscopy (cryo-TEM) (Figure S3).

Encapsulation of active components into the PDLLA-based stomatocyte cavity is a challenging task. One example where this is most apparent is the covalent approach adopted by the group of Wilson to localize enzymes in the stomatocytes' cavity; ${ }^{35}$ however, controlling such complex, multistep, functionalization steps remains elusive and necessitates alternative strategies. Inspired by previous examples showing the controlled growth of inorganic nanoparticles as an effective strategy for their inclusion in organic materials (e.g., protein and polymer cages), ${ }^{44}$ we developed a non-covalent approach based on the compartmentalized synthesis of inorganic $\mathrm{MnO}_{2}$ nanoparticles (MnPs) within the organic stomatocytes as an effective one-pot pathway for catalyst loading and subsequent construction of hybrid nanomotors. As evidenced by TEM tomographic analysis, our approach exploited the unique structural features of stomatocytes by utilizing their inner nanocompartment as a $3 \mathrm{D}$ template for the synthesis of inorganic MnPs that would be more readily entrapped within this domain and not escape through the $94 \mathrm{~nm}$ neck (Figures $1 \mathrm{E}$ and S4 and Videos S1 and S2). This compartmentalized synthesis of $\mathrm{MnPs}$ was achieved by dissolving potassium permanganate $\left(\mathrm{KMnO}_{4}\right)$ in the stomatocyte solution for $1 \mathrm{~h}$ prior to reduction using sodium thiosulfate $\left(\mathrm{Na}_{2} \mathrm{~S}_{2} \mathrm{O}_{3}\right)$. BSA was added to stabilize the resulting colloidal dispersion and to prevent uncontrolled aggregation. In this way, $\mathrm{MnPs}$ were formed and compartmentalized within the stomatocyte, whereas non-entrapped particles were removed readily using centrifugation (Figures 1B,D, S5, and S6). It is noteworthy that diverse types of preformed nanoparticles, including MnPs, could not be physically encapsulated within the cavity of PDLLA stomatocytes, as observed in initial trials (Figure S7). Therefore, the in situ synthesis/growth of $\mathrm{MnO}_{2}$ nanoparticles within the stomatocyte cavity was necessary for the successful realization of our hybrid nanomotor system. Cryo-TEM imaging and $3 \mathrm{D}$ tomographic reconstruction confirmed the well-defined nature of the resulting hybrid architectures (Figures 1D,E, S4, and S5 and Videos S1 and S2). Interestingly, although the average size of individual MnPs was ca. $25 \mathrm{~nm}$ (Figure S8), fractionally smaller than the apparent size of the neck, the high density of particles inside the stomach seemed to create a retaining force to prevent their escape, as observed from images from cryo-TEM and tomographic analysis (Figures 1E, S4, and S5 and Videos S1 and S2). Analysis of tomography data indicated the formation of nanoaggregates/agglomerates (i.e., particles attached to each other) with sizes larger than the neck of the stomatocytes (Figures $1 \mathrm{C}$ and S4 and Videos S1 and S2). Therefore, escape of such nanoparticles from the inner cavity did not occur, even after 3 months. Remarkably, the size of such nanoaggregates was significantly larger than that of the nanoparticles grown outside the stomatocytes. This can be attributed to a templating effect: the stomatocytes' nanocavity templates the growth of the $\mathrm{MnO}_{2}$ nanoparticles. This same effect was previously observed in the case of either in situ-grown nanoparticles or the assembly of enzymes in polystyrenebased stomatocytes. ${ }^{30,31,45}$ Such a difference in the sizes of nanoparticles formed inside and outside of the stomatocytes can be due to the difference in nanoparticle growth rate. The low volume of the stomatocyte nanocavity compared with the outer solution could result in the formation of fewer nucleation centers and hence larger particles. This is in agreement with previous reports that demonstrate the relationship between nanoparticle size and the concentration of both the inorganic salt and capping agent. ${ }^{45}$ This approach is general and would be applicable to the compartmentalized synthesis of a range of metal/metal oxide nanoparticles, with the open neck permitting the flux of molecular substrates, preventing coalescence/aggregation while facilitating application in processing methods such as microfluidics because of the overall size of the compartment.

In order to study the ability of our hybrid stomatocytes to undergo autonomous motion, NTA was used to record the nanomotors' movement and track their trajectories as MnPs catalyzed the formation of $\mathrm{O}_{2}$. In general, $\mathrm{O}_{2}$-driven propulsion is mediated by fuel decomposition and subsequent formation of nanobubbles that propel the particle through their release from the motor's chassis. The asymmetric structure of stomatocytes, with a unidirectional neck, are particularly suited 

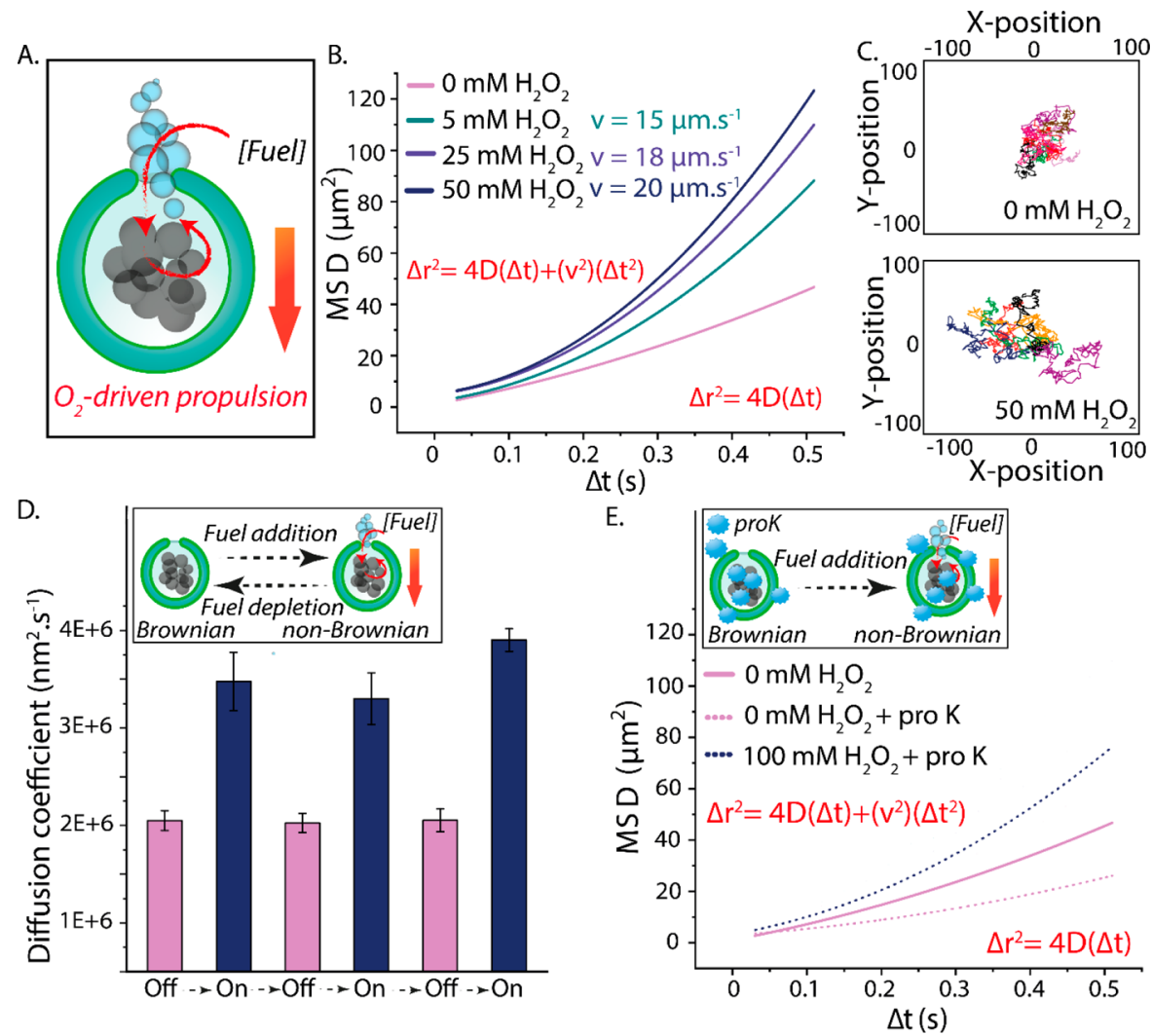

Figure 2. (A) Schematic demonstrating the spatial positioning of catalytic MnPs within stomatocytes, MnPs' capacity to convert a range of fuel concentrations into $\mathrm{O}_{2}$ nanobubbles, and the (hypothesized) mechanism by which motion occurs. (B) MSD and velocity of hybrid nanomotors in the presence of a range of $\mathrm{H}_{2} \mathrm{O}_{2}$ concentrations. The velocities were theoretically calculated from MSD $=(4 D) \Delta t+\left(v^{2}\right)\left(\Delta t^{2}\right)$. NTA measurements were performed by diluting MnP-loaded stomatocytes $(1: 1000)$ in $1 \times \mathrm{PBS}$ ([polymer] $=5 \mu \mathrm{g} \mathrm{mL}{ }^{-1}$ ). Thereafter, aliquots of $\mathrm{H}_{2} \mathrm{O}_{2}$ were added $($ to reach a final concentration of 5, 25, or $50 \mathrm{mM}$ ). (C) Tracked Brownian and non-Brownian trajectories in the absence and presence of fuel, respectively. (D) Diffusion coefficients of MnP-loaded stomatocytes as a result of multiple cycles of fuel addition and depletion. In order to ensure complete depletion of fuel, the time interval between experiments (fuel addition) was 1 day. (E) MSDs of hybrid stomatocytes exposed to $50 \mu \mathrm{g}$ $\mathrm{mL}^{-1}$ proteinase $\mathrm{K}$ (dotted line).

to exploit this bubble-propelled motion. This is indeed reflected by their propulsion, which was found to be proportional to the concentration of $\mathrm{H}_{2} \mathrm{O}_{2}$ (Figure $2 \mathrm{~A}$ and Video S3). The mean square displacements (MSDs) and velocities of such hybrid stomatocyte nanomotors were analyzed using Golestanian's self-diffusiophoretic model (the detailed analysis is described in SI section S2). ${ }^{46}$ The range of observed MSD values for hybrid stomatocytes upon addition of an increasing concentration of fuel reflects the inherent polydispersity in terms of $\mathrm{MnO}_{2}$ loading achieved in this system (Figure S9). Although one would expect linear fits of the MSD curves at relatively high $\Delta t$ (because of the short selfrotation time $\tau_{\mathrm{r}}$ of such small nanoparticles), nonlinear fits were identified at high concentrations of fuel (Figure 2B). Indeed, analysis of the anomalous diffusive exponent $(\alpha)$ value after addition of fuel showed an increase, which suggests an enhanced superdiffusion (propulsive) behavior (Figure S10). These results suggest that the autonomous movement of such nanomotors is a consequence of the bubble generation mechanism, which counteracts, to a certain extent, the effect of the short $\tau_{\mathrm{r}}$. Nevertheless, effects of such a short $\tau_{\mathrm{r}}$ on the motors' motion can be clearly seen in the shape of the nondirectional trajectories (Figure 2C). In order to confirm that the autonomous motion is a result of the catalytic process that takes place within the nanomotor cavity, control experiments were performed. First, a high concentration of
$\mathrm{H}_{2} \mathrm{O}_{2}$ (100 mM) was added to unloaded stomatocytes. This did not result in any autonomous motion, and all of the stomatocytes maintained their Brownian motion (Figure S11A and Video S4). Further controls in which empty stomatocytes were combined with free MnPs in solution yielded no motion, indicating the importance of catalyst spatial positioning within the stomatocytes (Figure S11B and Video S5).

To highlight the robust nature of these nanomotors, we studied their performance over time. To do so, nanomotors were tested on their ability to be reactivated upon fuel depletion (Figure 2D). After 3 days and over the course of three cycles of fuel addition and depletion, no change in their diffusion coefficient was recorded (as measured by NTA), indicating the stability of this hybrid nanomotor system and that the MnP platform is a highly suitable and robust catalytic nanomachine that is not degraded after a single use. Finally, the motility of our hybrid nanomotors was tested in a proteolytic environment, namely, in the presence of proteinase $\mathrm{K}$ at a concentration of $50 \mu \mathrm{g} \mathrm{mL}^{-1}\left(30\right.$ units $\left.\mathrm{mg}^{-1}\right)$. At first sight, interestingly, it was clear that the Brownian motion of the stomatocyte nanomotors in the absence of fuel was impacted by the presence of proteinase $\mathrm{K}$, with a ca. 2-fold lower MSD (Figure 2E). This is likely due to normal protein interaction with the stomatocytes' surface, which leads to an increase in the overall particle size and thus slower Brownian motion (as evidenced by the ca. $150 \mathrm{~nm}$ increase in 
hydrodynamic size; Figure S12). This hypothesis was also confirmed by measurement of the MSD of empty carriers in the presence of proteinase $\mathrm{K}$ at the same concentration (50 $\mu \mathrm{M}$ ), where a similar effect was observed (Figure S13). Despite this, the nanomotors' autonomous motion was clearly maintained in the presence of fuel, and only a slight reduction in the MSD was observed (compared to measurements without free proteins), highlighting the stability of this system and its relevance for application in a biological context. Taken together, these studies demonstrate the robust properties of our hybrid nanomotor compared with the more sensitive catalysts generally used in nanoscale motile systems.

To assess the potential of hybrid nanomotors for application in biomedical research, we first demonstrated the degradation of the $\mathrm{MnP}$ catalyst by glutathione (GSH), an intracellular antioxidant. In general, GSH is present at micromolar concentrations within the plasma, although this increases to millimolar levels within the cell. At high concentrations of GSH (10 mM), a significant color change was directly observed within $1 \mathrm{~min}$ (Figure 3A). The degradation of the $\mathrm{MnP}$ catalyst was also confirmed by the nanomotors' deactivation and consequent recovery of their Brownian motion upon GSH addition (Figure 3B). Motor activity under less severe conditions (10 $\mu \mathrm{M}$ GSH) was not affected in the same time frame (Figure S15). These studies indicated that under certain intracellular conditions (e.g., elevated reductant concentration) nanomotors would be deactivated. In fact, developing glutathione-responsive systems has been regarded as an appealing strategy for targeted drug delivery in cancer cells. ${ }^{47,48}$ Taking into account the potential use of nanomotors as drug nanocarriers, this would be advantageous, as such systems would have autonomous motion in the extracellular environments but be degraded at the target.

In our final set of experiments, we performed cellular testing using nanomotors to assess their biocompatibility and ability to function in a cellular environment. Particle toxicity was measured using HeLa cells treated with empty and loaded stomatocytes up to $250 \mu \mathrm{g} \mathrm{mL}^{-1}$ for $24 \mathrm{~h}$ (Figure $4 \mathrm{~A}$ and Table S2). Relatively low toxicity was observed for hybrid nanomotors at $250 \mu \mathrm{g} \mathrm{mL} \mathrm{m}^{-1}$ in comparison with other nanomotor systems that displayed similar (ca. 80\%) viability at lower concentrations. ${ }^{49}$ When cells were treated with toxic concentrations of $\mathrm{H}_{2} \mathrm{O}_{2}$ of either 125 or $250 \mu \mathrm{M}$ in the presence of unloaded stomatocytes, no population of viable cells was visible (Figure S16). To assess the ability of nanomotors to function under such conditions of low fuel levels, we conducted additional motion studies at $\mathrm{H}_{2} \mathrm{O}_{2}$ concentrations equivalent to those employed in the cell studies (i.e., 125 and $250 \mu \mathrm{M}$ ). Under such conditions, enhanced diffusion was observed, and the hybrid nanomotors retained their motile properties (Figure $\mathrm{S} 17$ ). $\mathrm{H}_{2} \mathrm{O}_{2}$ is a cytotoxic species that is generated at increasing concentrations in the tumor microenvironment and in cancerous cells; for instance, Guo and co-workers determined $\mathrm{H}_{2} \mathrm{O}_{2}$ levels of around 715 $\mu \mathrm{M}$ in bladder cancer tissues. ${ }^{41}$ Because of their ability to scavenge $\mathrm{H}_{2} \mathrm{O}_{2}$, harnessing it for their enhanced diffusion, nanomotors were utilized to rescue the viability of cells during exposure to this harmful oxidative species. Indeed, around 50\% viability was obtained after treatment of cells (exposed to 125 $\mu \mathrm{M} \mathrm{H}_{2} \mathrm{O}_{2}$ ) with nanomotors at $125 \mu \mathrm{g} \mathrm{mL}^{-1}$ (Figure $4 \mathrm{~B}$ and Table S3). This significantly increased to ca. $80 \%$ when the nanomotor concentration was elevated to $250 \mu \mathrm{g} \mathrm{mL}^{-1}$, near the background level as presented in Figure 4A. At an elevated
A. Catalyst Degradation

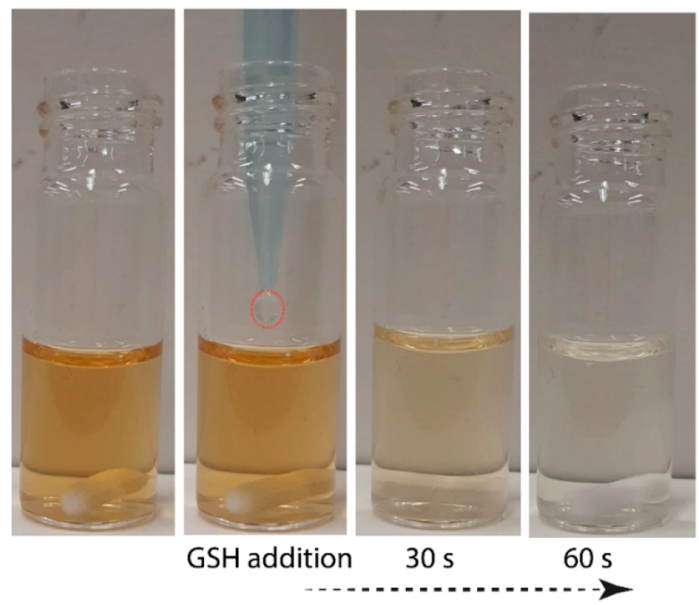

B. Motor Deactivation

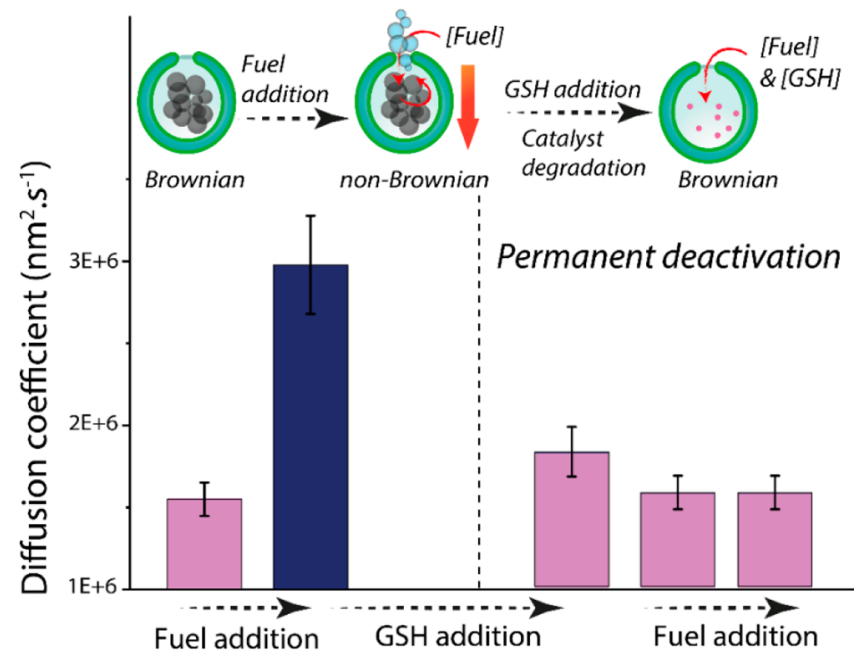

Figure 3. (A) Visual of $\mathrm{MnO}_{2}$ catalyst $(\mathrm{MnP})$ reduction by addition of high levels of glutathione (GSH) $(10 \mathrm{mM})$ to a 10 -fold dilution of $\mathrm{MnPs}$ in $1 \times \mathrm{PBS}$, where decoloration within $1 \mathrm{~min}$ indicates dissolution of the MnP catalyst. (B) Diffusion coefficients for fueled nanomotors before and after GSH addition, showing motor deactivation at high levels of GSH even after repeated fuel addition. Fuel was added at a final concentration of $100 \mathrm{mM}$. The diffusion coefficient for passive particles is lower than those presented in Figure 2 because of batch-to-batch differences in size distribution (Figure S14).

$\mathrm{H}_{2} \mathrm{O}_{2}$ concentration of $250 \mu \mathrm{M}$, cell viability recovered up to ca. $45 \%$ because of the performance limits of the nanomotor. To validate that the nanomotor-mediated increase in cellular viability was commensurate with decreasing reactive oxygen species (ROS) levels, additional experiments were performed using a fluorescent ROS indicator (CM-H2DCFDA). Confocal microscopy (and quantitative analysis) confirmed that under the most extreme conditions $\left(250 \mu \mathrm{M} / 250 \mu \mathrm{g} \mathrm{mL}^{-1}\right)$, cellular ROS was significantly reduced in the presence of hybrid nanomotors compared with unloaded stomatocytes, which did not have a significant effect (Figures 4C-E, S18, and S19). Although this effect was very pronounced, this only correlated to a partial recovery in viability, as shown in Figure 4B.

As a proof-of-principle experiment, we compared the penetration behavior of fluorescently labeled hybrid nanomotors and empty stomatocytes in 3D cell spheroids (the 

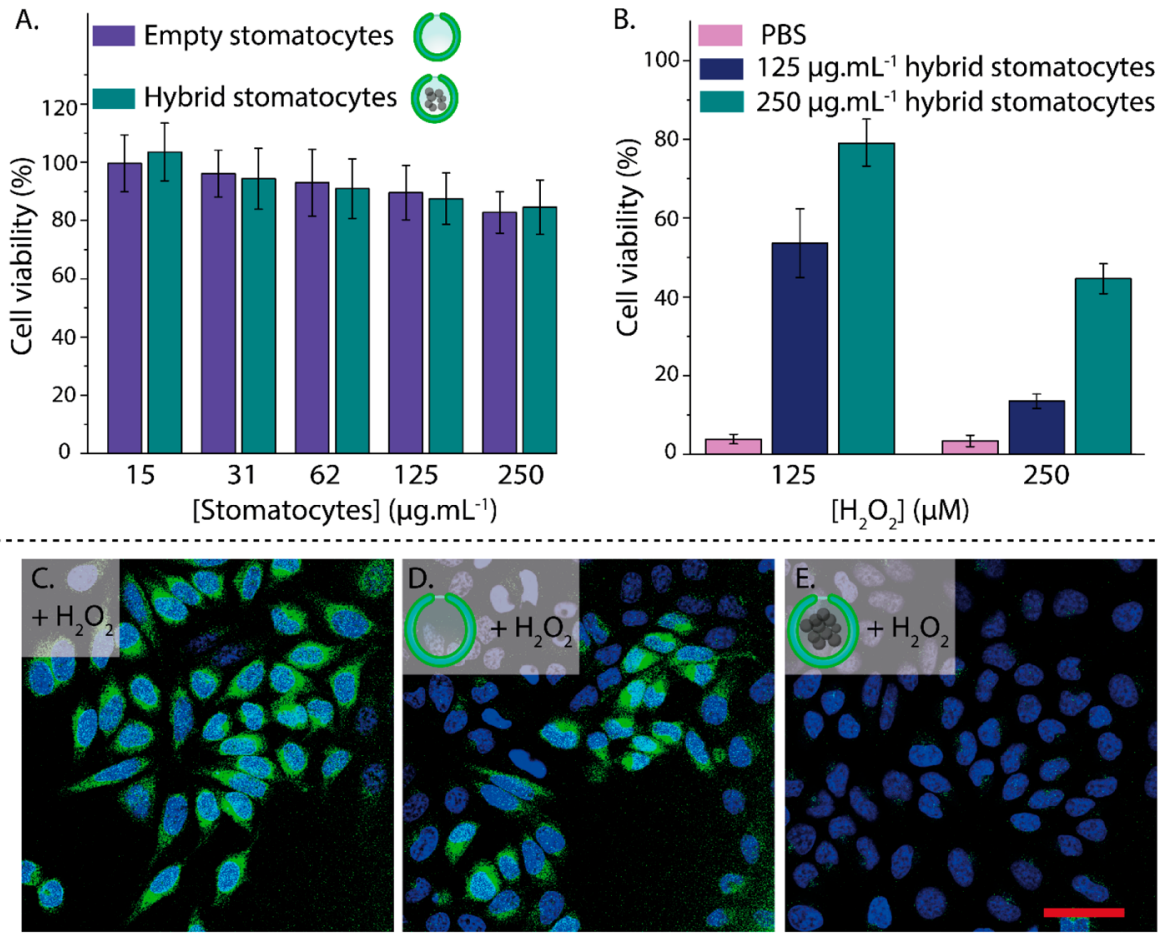

Empty stomatocytes
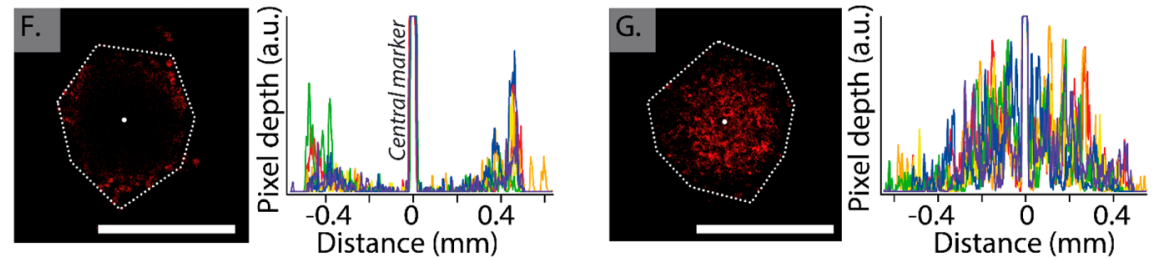

Figure 4. (A) Viability of HeLa cells after exposure to empty and MnP-loaded stomatocytes at a range of concentrations $\left(15-250 \mu \mathrm{g} \mathrm{mL}^{-1}\right)$ as determined by MTT assay. (B) Hybrid stomatocytes rescue the viability of HeLa cells after coincubation with $\mathrm{H}_{2} \mathrm{O}_{2}$. (C) ROS induction (as indicated by the green fluorescence of CM-H2DCFDA stain) in HeLa cells after treatment with $\mathrm{H}_{2} \mathrm{O}_{2}$ is significantly reduced by the presence of hybrid nanomotors (blue $=$ Hoechst nuclear stain; scale bar $=50 \mu \mathrm{m})$. (F) Penetration of empty stomatocytes and (G) hybrid nanomotors into 3D cell spheroids (coincubated with $250 \mu \mathrm{M} \mathrm{H}_{2} \mathrm{O}_{2}$ ) with accompanying line profiles for comparison (experiments were replicated in triplicate, cf. Figures S21 and S22; scale bars $=1 \mathrm{~mm}$ ).

experiments were conducted in triplicate using $250 \mu \mathrm{M} \mathrm{H}_{2} \mathrm{O}_{2}$; Figures 4F,G and S20-S22). A nuclear cell stain (Hoechst) was used to provide an indication of overall spheroid morphology, where treatment with $250 \mu \mathrm{M} \mathrm{H}_{2} \mathrm{O}_{2}$ did not compromise cell stability (compared with those cultured in two dimensions), in agreement with the literature that reports higher toxicity resistance of 3D cell models compared with 2D ones (Figure S20). ${ }^{50,51}$ We observed that nanomotors (fueled by $\mathrm{H}_{2} \mathrm{O}_{2}$ ) penetrated effectively into the spheroid, whereas control particles showed significantly reduced infiltration of the dense cellular framework. These data highlight the applicability of our technology and provide proof-of-principle results to guide future biomedical studies.

In summary, we have presented a new strategy for the fabrication of biodegradable hybrid stomatocyte nanomotors. The unique structural features of such stomatocytes were utilized to template the in situ one-pot preparation of a biodegradable $\mathrm{MnO}_{2}$ catalytic core through a process of compartmentalized synthesis. Such hybrid nanomotors show autonomous motion in the presence of fuel and maintain their stability through extended periods of time. Moreover, such nanomotors maintained their activity in physiological medium and are highly biocompatible-able to reduce local ROS levels to protect cells. This system has strong potential in biomedical research and highlights the capacity of molecular engineering for the creation of morphologically diverse, functional nanosystems. To further showcase this application, we conducted proof-of-principle experiments to demonstrate effective penetration of nanomotors into $3 \mathrm{D}$ cell spheroids. Future research will engage the utility of compartmentalized inorganic nanocatalysts (and assorted stabilizing molecules) to drive autonomous (nano)motion and explore their biorelated applications. We believe that our approach can offer a general route toward assembling functional nanomotors and paves the way to overcome limitations associated with traditional physical encapsulation of active catalysts. Further studies will expand the range of nanoparticles that can be integrated within stomatocyte motors and explore in more detail the mechanism of formation.

\section{ASSOCIATED CONTENT}

\section{Supporting Information}

The Supporting Information is available free of charge at https://pubs.acs.org/doi/10.1021/acs.nanolett.0c01268.

Experimental procedures and supporting tables and figures (PDF) 
Surface view from cryo-TEM tomography of $\mathrm{MnP}$ loaded stomatocytes showing the overall structure of the nanomotor (MP4)

Cryo-TEM tomography of MnP-loaded stomatocytes showing the structure of the $\mathrm{MnO}_{2}$ nanoparticles inside the cavity of the nanomotor (MP4)

Compilation of NTA videos of motile MnP-loaded stomatocytes in the presence of fuel (MP4)

NTA video of empty stomatocytes in the presence of fuel (MP4)

NTA video of empty stomatocytes and free MnPs in the presence of fuel(MP4)

\section{AUTHOR INFORMATION}

\section{Corresponding Authors}

Loai K. E. A. Abdelmohsen - Department of Bio-Organic Chemistry, Institute of Complex Molecular Systems (ICMS), Eindhoven University of Technology, 5600 MB Eindhoven, The Netherlands; 10 orcid.org/0000-0002-0094-1893; Email: 1.k.e.a.abdelmohsen@tue.nl

Jan C. M. van Hest - Department of Bio-Organic Chemistry, Institute of Complex Molecular Systems (ICMS), Eindhoven University of Technology, 5600 MB Eindhoven, The Netherlands; 10 orcid.org/0000-0001-7973-2404; Email: j.c.m.v.hest@tue.nl

\section{Authors}

Imke A. B. Pijpers - Department of Bio-Organic Chemistry, Institute of Complex Molecular Systems (ICMS), Eindhoven University of Technology, 5600 MB Eindhoven, The Netherlands

Shoupeng Cao - Department of Bio-Organic Chemistry, Institute of Complex Molecular Systems (ICMS), Eindhoven University of Technology, 5600 MB Eindhoven, The Netherlands

Antoni Llopis-Lorente - Department of Bio-Organic Chemistry, Institute of Complex Molecular Systems (ICMS), Eindhoven University of Technology, 5600 MB Eindhoven, The Netherlands

Jianzhi Zhu - Department of Bio-Organic Chemistry, Institute of Complex Molecular Systems (ICMS), Eindhoven University of Technology, 5600 MB Eindhoven, The Netherlands

Shidong Song - Department of Bio-Organic Chemistry, Institute of Complex Molecular Systems (ICMS), Eindhoven University of Technology, 5600 MB Eindhoven, The Netherlands

Rick R. M. Joosten - Center for Multiscale Electron Microscopy (CMEM), Eindhoven University of Technology, $5600 \mathrm{MB}$ Eindhoven, The Netherlands

Fenghua Meng - Biomedical Polymers Laboratory, College of Chemistry, Chemical Engineering and Materials Science, Soochow University, Suzhou 215123, P. R. China; (1) orcid.org/0000-0002-8608-7738

Heiner Friedrich - Center for Multiscale Electron Microscopy (CMEM) and Department of Chemical Engineering and Chemistry, Physical Chemistry, Institute of Complex Molecular Systems (ICMS), Eindhoven University of Technology, 5600 MB Eindhoven, The Netherlands; 10 orcid.org/0000-00034582-0064

David S. Williams - Department of Chemistry, College of Science, Swansea University, Swansea SA2 8PP, U.K.; (1) orcid.org/0000-0002-8209-6899
Samuel Sánchez - Institute for Bioengineering of Catalonia (IBEC), The Barcelona Institute of Science and Technology, 08028 Barcelona, Spain; 이이.org/0000-0002-5845-8941

Complete contact information is available at: https://pubs.acs.org/10.1021/acs.nanolett.0c01268

\section{Author Contributions}

I.A.B.P., J.C.M.v.H., and L.K.E.A.A. designed the project. I.A.B.P. conducted the synthesis, characterization, and motility experiments. S.C. performed the in vitro cell studies. R.R.M.J. and H.F. performed the tomography experiments and the 3D rendering. J.C.M.v.H. and L.K.E.A.A. guided the research. I.A.B.P. and L.K.E.A.A. wrote the manuscript. All of the authors contributed to the critical discussion of the results and commented on the manuscript.

\section{Notes}

The authors declare no competing financial interest.

\section{ACKNOWLEDGMENTS}

The authors acknowledge the support from the Dutch Ministry of Education, Culture and Science (Gravitation Program 024.001.035) and NWO-NSFC Advanced Materials (Project 792.001.015). The authors acknowledge the support by the National Natural Science Foundation of China (51561135010). We thank the Spanish MINECO (BOTSinFluids Project), the Foundation BBVA (MEDIROBOTS Project), and the CERCA Program of the Generalitat de Catalunya. We thank the Ser Cymru II Programme for support of D.S.W.; this project received funding from the European Union's Horizon 2020 Research and Innovation Programme under Marie Skłodowska-Curie Grant Agreement 663830.

\section{REFERENCES}

(1) Wang, H.; Pumera, M. Fabrication of Micro/Nanoscale Motors. Chem. Rev. 2015, 115 (16), 8704-8735.

(2) Gao, W.; Wang, J. Synthetic Micro/Nanomotors in Drug Delivery. Nanoscale 2014, 6 (18), 10486-10494.

(3) Lee, T. C.; Alarcón-Correa, M.; Miksch, C.; Hahn, K.; Gibbs, J. G.; Fischer, P. Self-Propelling Nanomotors in the Presence of Strong Brownian Forces. Nano Lett. 2014, 14 (5), 2407-2412.

(4) Das, S.; Shklyaev, O. E.; Altemose, A.; Shum, H.; Ortiz-Rivera, I.; Valdez, L.; Mallouk, T. E.; Balazs, A. C.; Sen, A. Harnessing Catalytic Pumps for Directional Delivery of Microparticles in Microchambers. Nat. Commun. 2017, 8, 14384.

(5) Sanchez, S.; Soler, L.; Katuri, J. Chemically Powered Micro- and Nanomotors. Angew. Chem., Int. Ed. 2015, 54 (5), 1414-1444.

(6) Brooks, A. M.; Tasinkevych, M.; Sabrina, S.; Velegol, D.; Sen, A.; Bishop, K. J. M. Shape-Directed Rotation of Homogeneous Micromotors via Catalytic Self-Electrophoresis. Nat. Commun. 2019, $10(1), 495$.

(7) Dey, K. K.; Sen, A. Chemically Propelled Molecules and Machines. J. Am. Chem. Soc. 2017, 139 (23), 7666-7676.

(8) Schattling, P.; Thingholm, B.; Städler, B. Enhanced Diffusion of Glucose-Fueled Janus Particles. Chem. Mater. 2015, 27 (21), 74127418.

(9) Gao, W.; Kagan, D.; Pak, O. S.; Clawson, C.; Campuzano, S.; Chuluun-Erdene, E.; Shipton, E.; Fullerton, E. E.; Zhang, L.; Lauga, E.; Wang, J. Cargo-Towing Fuel-Free Magnetic Nanoswimmers for Targeted Drug Delivery. Small 2012, 8 (3), 460-467.

(10) Xu, D.; Wang, Y.; Liang, C.; You, Y.; Sanchez, S.; Ma, X. SelfPropelled Micro/Nanomotors for On-Demand Biomedical Cargo Transportation. Small 2019, 1902464.

(11) Llopis-Lorente, A.; Garciá-Fernández, A.; Murillo-Cremaes, N.; Hortelaõ, A. C.; Patinõ, T.; Villalonga, R.; Sancenón, F.; MartínezMáñez, R.; Sánchez, S. Enzyme-Powered Gated Mesoporous Silica 
Nanomotors for on-Command Intracellular Payload Delivery. ACS Nano 2019, 13 (10), 12171-12183.

(12) Villa, K.; Pumera, M. Fuel-Free Light-Driven Micro/Nanomachines: Artificial Active Matter Mimicking Nature. Chem. Soc. Rev. 2019, 48 (19), 4966-4978.

(13) Shao, J.; Abdelghani, M.; Shen, G.; Cao, S.; Williams, D. S.; Van Hest, J. C. M. Erythrocyte Membrane Modified Janus Polymeric Motors for Thrombus Therapy. ACS Nano 2018, 12 (5), 4877-4885.

(14) Liu, L.; Chen, B.; Liu, K.; Gao, J.; Ye, Y.; Wang, Z.; Qin, N.; Wilson, D. A.; Tu, Y.; Peng, F. Wireless Manipulation of Magnetic/ Piezoelectric Micromotors for Precise Neural Stem-Like Cell Stimulation. Adv. Funct. Mater. 2020, 30, 1910108.

(15) Chen, X. Z.; Liu, J. H.; Dong, M.; Müller, L.; Chatzipirpiridis, G.; Hu, C.; Terzopoulou, A.; Torlakcik, H.; Wang, X.; Mushtaq, F.; Puigmartí-Luis, J.; Shen, Q. D.; Nelson, B. J.; Pané, S. Magnetically Driven Piezoelectric Soft Microswimmers for Neuron-like Cell Delivery and Neuronal Differentiation. Mater. Horiz. 2019, 6 (7), $1512-1516$

(16) Xuan, M.; Shao, J.; Gao, C.; Wang, W.; Dai, L.; He, Q. SelfPropelled Nanomotors for Thermomechanically Percolating Cell Membranes. Angew. Chem., Int. Ed. 2018, 57 (38), 12463-12467.

(17) Patino, T.; Porchetta, A.; Jannasch, A.; Lladó, A.; Stumpp, T.; Schäffer, E.; Ricci, F.; Sánchez, S. Self-Sensing Enzyme-Powered Micromotors Equipped with PH-Responsive DNA Nanoswitches. Nano Lett. 2019, 19 (6), 3440-3447.

(18) Wan, M.; Chen, H.; Wang, Q.; Niu, Q.; Xu, P.; Yu, Y.; Zhu, T.; Mao, C.; Shen, J. Bio-Inspired Nitric-Oxide-Driven Nanomotor. Nat. Commun. 2019, 10, 966.

(19) Mushtaq, F.; Torlakcik, H.; Hoop, M.; Jang, B.; Carlson, F.; Grunow, T.; Läubli, N.; Ferreira, A.; Chen, X. Z.; Nelson, B. J.; Pané, S. Motile Piezoelectric Nanoeels for Targeted Drug Delivery. Adv. Funct. Mater. 2019, 29 (12), 1808135.

(20) Wu, Z.; Lin, X.; Si, T.; He, Q. Recent Progress on Bioinspired Self-Propelled Micro/Nanomotors via Controlled Molecular SelfAssembly. Small 2016, 12 (23), 3080-3093.

(21) Gao, W.; de Ávila, B. E. F.; Zhang, L.; Wang, J. Targeting and Isolation of Cancer Cells Using Micro/Nanomotors. Adv. Drug Delivery Rev. 2018, 125, 94-101.

(22) Wu, Z.; Troll, J.; Jeong, H. H.; Wei, Q.; Stang, M.; Ziemssen, F.; Wang, Z.; Dong, M.; Schnichels, S.; Qiu, T.; Fischer, P. A Swarm of Slippery Micropropellers Penetrates the Vitreous Body of the Eye. Sci. Adv. 2018, 4 (11), No. eaat4388.

(23) Hortelao, A. C.; Carrascosa, R.; Murillo-Cremaes, N.; Patiño, T.; Sánchez, S. Targeting 3D Bladder Cancer Spheroids with UreasePowered Nanomotors. ACS Nano 2019, 13 (1), 429-439.

(24) Ramos-Docampo, M. A.; Fernández-Medina, M.; Taipaleenmäki, E.; Hovorka, O.; Salgueiriño, V.; Städler, B. Microswimmers with Heat Delivery Capacity for 3D Cell Spheroid Penetration. ACS Nano 2019, 13 (10), 12192-12205.

(25) Somasundar, A.; Ghosh, S.; Mohajerani, F.; Massenburg, L. N.; Yang, T.; Cremer, P. S.; Velegol, D.; Sen, A. Positive and Negative Chemotaxis of Enzyme-Coated Liposome Motors. Nat. Nanotechnol. 2019, 14 (12), 1129-1134.

(26) Sun, T.; Zhang, Y. S.; Pang, B.; Hyun, D. C.; Yang, M.; Xia, Y. Engineered Nanoparticles for Drug Delivery in Cancer Therapy. Angew. Chem., Int. Ed. 2014, 53 (46), 12320-12364.

(27) Ariga, K.; Leong, D. T.; Mori, T. Nanoarchitectonics for Hybrid and Related Materials for Bio-Oriented Applications. Adv. Funct. Mater. 2018, 28 (27), 1702905.

(28) Kim, K. T.; Zhu, J.; Meeuwissen, S. A.; Cornelissen, J. J. L. M.; Pochan, D. J.; Nolte, R. J. M.; Van Hest, J. C. M. Polymersome Stomatocytes: Controlled Shape Transformation in Polymer Vesicles. J. Am. Chem. Soc. 2010, 132 (36), 12522-12524.

(29) Rikken, R. S. M.; Engelkamp, H.; Nolte, R. J. M.; Maan, J. C.; Van Hest, J. C. M.; Wilson, D. A.; Christianen, P. C. M. Shaping Polymersomes into Predictable Morphologies via Out-of-Equilibrium Self-Assembly. Nat. Commun. 2016, 7, 12606.

(30) Abdelmohsen, L. K. E. A.; Nijemeisland, M.; Pawar, G. M.; Janssen, G. J. A.; Nolte, R. J. M.; Van Hest, J. C. M.; Wilson, D. A.
Dynamic Loading and Unloading of Proteins in Polymeric Stomatocytes: Formation of an Enzyme-Loaded Supramolecular Nanomotor. ACS Nano 2016, 10 (2), 2652-2660.

(31) Nijemeisland, M.; Abdelmohsen, L. K. E. A.; Huck, W. T. S.; Wilson, D. A.; Van Hest, J. C. M. A Compartmentalized Out-ofEquilibrium Enzymatic Reaction Network for Sustained Autonomous Movement. ACS Cent. Sci. 2016, 2 (11), 843-849.

(32) Abdelmohsen, L. K. E. A.; Williams, D. S.; Pille, J.; Ozel, S. G.; Rikken, R. S. M.; Wilson, D. A.; Van Hest, J. C. M. Formation of Well-Defined, Functional Nanotubes via Osmotically Induced Shape Transformation of Biodegradable Polymersomes. J. Am. Chem. Soc. 2016, 138 (30), 9353-9356.

(33) Pijpers, I. A. B.; Abdelmohsen, L. K. E. A.; Williams, D. S.; Van Hest, J. C. M. Morphology under Control: Engineering Biodegradable Stomatocytes. ACS Macro Lett. 2017, 6 (11), 1217-1222.

(34) Shao, J.; Pijpers, I. A. B.; Cao, S.; Williams, D. S.; Yan, X.; Li, J.; Abdelmohsen, L. K. E. A.; van Hest, J. C. M. Biomorphic Engineering of Multifunctional Polylactide Stomatocytes toward Therapeutic Nano-Red Blood Cells. Adv. Sci. 2019, 6 (5), 1801678.

(35) Toebes, B. J.; Cao, F.; Wilson, D. A. Spatial Control over Catalyst Positioning on Biodegradable Polymeric Nanomotors. Nat. Commun. 2019, 10, 5308.

(36) Zhao, Z.; Fan, H.; Zhou, G.; Bai, H.; Liang, H.; Wang, R.; Zhang, X.; Tan, W. Activatable Fluorescence/MRI Bimodal Platform for Tumor Cell via $\mathrm{MnO}_{2}$ Nanosheet-Aptamer Nanoprobe. J. Am. Chem. Soc. 2014, 136 (32), 11220-11223.

(37) Yang, G.; Xu, L.; Chao, Y.; Xu, J.; Sun, X.; Wu, Y.; Peng, R.; Liu, Z. Hollow $\mathrm{MnO}_{2}$ as a Tumor-Microenvironment-Responsive Biodegradable Nano-Platform for Combination Therapy Favoring Antitumor Immune Responses. Nat. Commun. 2017, 8, 902.

(38) Song, M.; Liu, T.; Shi, C.; Zhang, X.; Chen, X. Bioconjugated Manganese Dioxide Nanoparticles Enhance Chemotherapy Response by Priming Tumor-Associated Macrophages toward M1-like Phenotype and Attenuating Tumor Hypoxia. ACS Nano 2016, 10 (1), 633-647.

(39) Liu, X.; Wang, Q.; Zhao, H.; Zhang, L.; Su, Y.; Lv, Y. BSATemplated $\mathrm{MnO}_{2}$ Nanoparticles as Both Peroxidase and Oxidase Mimics. Analyst 2012, 137 (19), 4552-4558.

(40) Baral, A.; Satish, L.; Das, D. P.; Sahoo, H.; Ghosh, M. K. Construing the Interactions between $\mathrm{MnO}_{2}$ Nanoparticle and Bovine Serum Albumin: Insight into the Structure and Stability of a ProteinNanoparticle Complex. New J. Chem. 2017, 41 (16), 8130-8139.

(41) Lin, T.; Zhao, X.; Zhao, S.; Yu, H.; Cao, W.; Chen, W.; Wei, $\mathrm{H}$.; Guo, H. $\mathrm{O}_{2}$-Generating $\mathrm{MnO}_{2}$ Nanoparticles for Enhanced Photodynamic Therapy of Bladder Cancer by Ameliorating Hypoxia. Theranostics 2018, 8 (4), 990-1004.

(42) Jiang, D.; Ni, D.; Rosenkrans, Z. T.; Huang, P.; Yan, X.; Cai, W. Nanozyme: New Horizons for Responsive Biomedical Applications. Chem. Soc. Rev. 2019, 48 (14), 3683-3704.

(43) Huang, Y.; Ren, J.; Qu, X. Nanozymes: Classification, Catalytic Mechanisms, Activity Regulation, and Applications. Chem. Rev. 2019, 119 (6), 4357-4412.

(44) Fan, Z.; Chen, X.; Köhn Serrano, M.; Schmalz, H.; Rosenfeldt, S.; Förster, S.; Agarwal, S.; Greiner, A. Polymer Cages as Universal Tools for the Precise Bottom-Up Synthesis of Metal Nanoparticles. Angew. Chem., Int. Ed. 2015, 54 (48), 14539-14544.

(45) Wilson, D. A.; Nolte, R. J. M.; van Hest, J. C. M. Entrapment of Metal Nanoparticles in Polymer Stomatocytes. J. Am. Chem. Soc. 2012, 134 (24), 9894-9897.

(46) Howse, J. R.; Jones, R. A. L.; Ryan, A. J.; Gough, T.; Vafabakhsh, R.; Golestanian, R. Self-Motile Colloidal Particles: From Directed Propulsion to Random Walk. Phys. Rev. Lett. 2007, 99 (4), 048102.

(47) Cheng, R.; Feng, F.; Meng, F.; Deng, C.; Feijen, J.; Zhong, Z. Glutathione-Responsive Nano-Vehicles as a Promising Platform for Targeted Intracellular Drug and Gene Delivery. J. Controlled Release 2011, 152 (1), 2-12.

(48) Han, L.; Zhang, X. Y.; Wang, Y. L.; Li, X.; Yang, X. H.; Huang, M.; Hu, K.; Li, L. H.; Wei, Y. Redox-Responsive Theranostic 
Nanoplatforms Based on Inorganic Nanomaterials. J. Controlled Release 2017, 259, 40-52.

(49) Sun, J.; Mathesh, M.; Li, W.; Wilson, D. A. Enzyme-Powered Nanomotors with Controlled Size for Biomedical Applications. ACS Nano 2019, 13 (9), 10191-10200.

(50) Godugu, C.; Patel, A. R.; Desai, U.; Andey, T.; Sams, A.; Singh, M. AlgiMatrix ${ }^{\mathrm{TM}}$ Based 3D Cell Culture System as an In-Vitro Tumor Model for Anticancer Studies. PLoS One 2013, 8 (1), No. e53708.

(51) Judée, F.; Fongia, C.; Ducommun, B.; Yousfi, M.; Lobjois, V.; Merbahi, N. Short and Long Time Effects of Low Temperature Plasma Activated Media on 3D Multicellular Tumor Spheroids. Sci. Rep. 2016, 6 (1), No. 21421. 